Research Square

\title{
PTPN9 Genes Polymorphism as a Protect Factor in Lumbar Disc Herniation Development
}

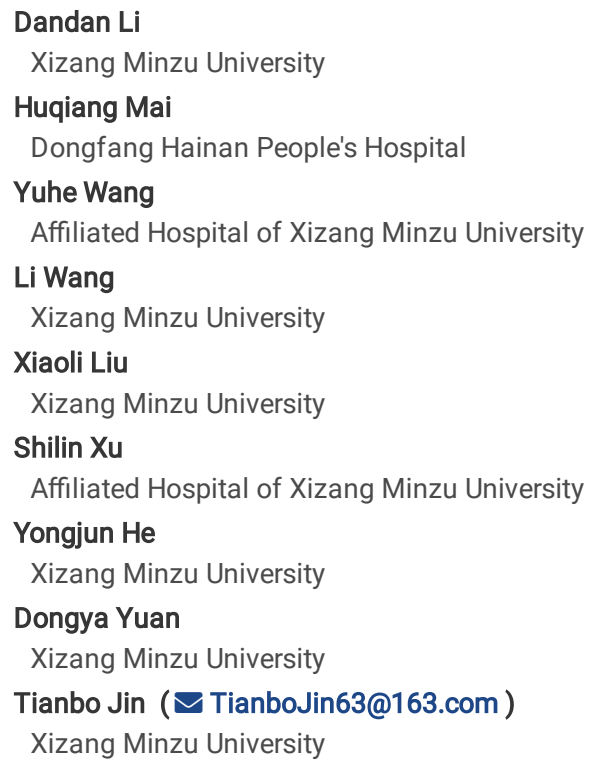




\section{Abstract}

Background: Lumbar disc herniation (LDH) is a high incidence spinal disease caused by disc degeneration, nuclear pulse displacement or fiber ring degeneration. This study aimed to explore the correlation between PTPN9 susceptibility and lumbar disc herniation in Chinese Han population.

Methods: The subjects were 504 patients with lumbar disc hernia and 503 controls. PTPN9 polymorphism (rs76107647, rs10851882, rs753992, rs11072552) are genotype using Agena MassArray. In addition, Logistic regression was used for odds ratios (ORs) and $95 \%$ confidence intervals (Cls) to assess the impact of genetic polymorphisms on LDH occurrence.

Results: In the allele model, rs76107647 A ( $p=0.023)$ is associated with a reduced risk of LDH. In the analysis of genetic models, we found that rs76107647 (adjusted, A/A vs. G/G: $p=0.035 ; A / A$ vs. AG/GG: $p=0.044$; log - additive model: $p=0.022$, respectively) and rs11072552 (adjusted, C/C vs. A/A: $p=0.048$; $C / C$ vs. CA/AA: $p=0.010$, respectively)were significantly related to $L D H$ risk reduction. After age stratification, rs76107647\adjusted, $A / A$ vs. $G / G: p=0.036$; A/G vs. $G / G: p=0.008$, ; AA/AG vs. G/G: $p=0.002$; log-additive model: $p=0.001$, respectively) can reduce the risk of LDH for young people $\leq 49$ years old, while rs11072552 (adjusted, CC vs. CA/AA: $p=0.009$ ) can significantly reduce the risk of LDH in patients over 49 years old. With gender stratification, rs76107647 (adjusted, A/A vs. G/G: $p=0.017$, ; A/A vs. AG/GG: $p=0.019$; log - additive model: $p=0.018$, respectively) was significantly associated with reduced LDH risk in female.

Conclusion: Our research suggests that PTPN9 polymorphisms may have protective effects on the risk of LDH in Chinese Han population.

\section{Introduction}

Lumbar disc herniation (LDH) is a spinal disease caused by degeneration of the intervertebral disc space, nuclear displacement, or circular fibrosis[1]. It is considered a worldwide health problem even though it is a benign disease. Because it can cause physical weakness, negatively affect work activities and the body, reduce the comfort of life, and cause distress in people's hearts[2]. About $90 \%$ of sciatica is caused by symptomatic LDH, and it is also the most common manifestation of symptomatic LDH[3]. Although mechanical deformation may lead to radiation-induced pain, it is currently believed that autoimmune response and related inflammatory factors are the major factors leading to this symptom[4]. Epidemiological studies have shown that smoking, age, gender, BMI, occupation type and physical activity are the main risk factors for lumbar disc degeneration and hernia[5]. Recent studies have shown that heritability plays a vital role in the process of LDH, and its heritability is $52 \%-68 \%$ [6]. Because LDH is often seen in adults and of course occurs in adolescents[7], understanding the relationship between LDH and genetics can give patients new preventive and therapeutic measures. The study of LDHrelated genes has laid a foundation for the interpretation of its genetics, and at the same time opened up a new world for LDH ethics research[8].

PTP-MEG2 (also known as PTPN9) is a type of non-selective PTP. It was first cloned from the cDNA library of human umbilical cord endothelial cells (HUVECs) and megakaryocytes, which is different from other PTP mammals, because there is an assumed lipid field at the end of $\mathrm{NH2}$.[9].It is a cytoplasmic protein whose function is mainly to regulate cell growth, cell cycle and malignant transformation[10]. Studies have shown that it plays an important role in multiple cancer processes. In breast cancer cells, PTP-Meg2 directly dephosphorylates EGFR and inhibits EGFR induced signal transduction[11]. Overexpressed in esophageal squamous cell carcinoma (ESCC) specimens, which indicates that PTPN9 may be an oncogene during ESCC[12]. Moreover, the expression of PTPN9 is down-regulated in human hepatocellular carcinoma (HCC) tumor tissues, and the decrease of PTPN9 expression is related to the deterioration of the overall survival of HCC patients. Its consumption inhibits apoptosis and promotes the proliferation of HCC cells[13]. In colorectal cancer, PTPN9 inhibits cell growth and survival by inhibiting the expression of Stat3, which suggests an important basic mechanism for regulating cell growth and provides a new target for the treatment of colorectal cancer[14]. In cervical cancer (CC), the decreased expression of PTPN9 is caused by the up-regulation of miR-613, which may promote the proliferation, invasion and migration of CC cells, revealing that the miR-613/PTPN9 axis may be a potential therapeutic target for the treatment of $\mathrm{CC}[15]$. At the same time, MEG2 is a tumor suppressor gene for gastric cancer, which is negatively regulated by miR-181a-5p[16]. In addition, MEG2-mediated secretory vesicles play an irreplaceable role in the development of neural tubes, blood vessels and bone, as well as the activation of mature platelets and lymphocytes[17], which shows that PTPN9 plays an important role in bone development. However, up to now, there is no study on the possible correlation between PTPN9 genes and LDH susceptibility in Chinese Han population.So we will explore the relationship between PTPN9 and LDH.

So, we conducted a case-control study to detect the relationship between the SNP and LDH of the single nucleotide polymorphisms in the genes of $P$ TPN9, and to explain their association with the risk of LDH in Chinese Han population. Identifying a positive finding is the purpose of this study and provides a basis for the treatment of LDH.

\section{Materials And Methods Study subjects}

504 patients with lumbar disc herniation (294 males, 210 females) and 503 healthy controls (294 males, 209 females) randomly selected from the Xi'an Red Cross Hospital from 2016 to 2017 the study. All the subjects were Chinese, and they were not related by blood. The criteria for selection as a patient are: first, the patient must have symptoms of lumbar pain and sciatica; secondly, the positive test of the lumbar spine straight leg lift is positive; finally, there is a positive reaction by CT or MRI of the lumbar spine. The typical manifestations of lumbar disc herniation are the following: (1) repeated low back pain; (2) radioactive sciatica; (3) numbness of one or both lower limbs; (4) compression of the cauda equina nerve, forming cauda equina syndrome. Depending on Pfrrmann grading system, lumbar MRI was diagnosed as lumbar disc herniation. This study excluded patients with tumors, autoimmune diseases, complex blood disorders, trauma, rheumatoid arthritis, as well as those with associated lumbar disease (including congenital dysplasia of the spine, intraspine tumors, lumbar spinal stenosis, lumbar spondylolisthesis ) [8]. Patients with one of the following conditions shall be excluded: (1) spondylitis, spondylolisthesis, osteoarthritis, acute or chronic inflammatory disease, history of diabetes or hypertension; (2) has taken or is taking immunosuppressive drugs; and (3) has 
received IVD operation[4]. All the subjects are Han Chinese, which can be traced back to at least three generations. Prior to the study, we obtained informed consent from all participants. This study is in line with the declaration of Helsinki and approved by the ethics committee of Tibet University for nationalities.

\section{Genotyping assay}

We used the venipuncture method to draw the fasting peripheral blood of the patient into the anticoagulation blood vessel and store it at $-80^{\circ} \mathrm{C}$. We used GoldMag-Mini Whole Blood Genomic DNA Purification Kit (GoldMag. Co. Ltd.) to extract DNA, following the manufacturer's agreement. Then the DNA concentration was determined by spectrophotometry (NanoDrop 2000). The rs76107647, rs 10851882 , rs753992 and rs 11072552 found in PTPN9 are based on our 1000 genome project data囚http://www.internationalgome.org/), and their micro allele frequency (MAF) exceeded 5\%. To design four SNP primers for amplification and extension, we used Agena Bioscience Assay Design Suite V2.0 software (https://agenacx.com/online-tools/). Multiple SNP MassEXTEND analysis was designed using Agena-MassARRAY analysis design 4.0 software, and according to the manufacturer's explanation, SNP genotyping was detected by Agena massarray RS1000. Data management and analysis were performed using Agena Typer 4.0 software. Then, we conducted haplotype analysis using Haploview 4.2 software.

\section{Statistical analysis}

We analyzed the data using SPSS 19.0 software (SPSS Inc., Chicago, IL). The Hardy-Weinberg equilibrium (HWE) test was performed on the genotype frequency of the control group by chi-square test, and the allele frequency and genotype frequency of each SNP in the two groups were tested by Pearson chisquare test or $\mathrm{t}$ test, so as to determine the association between genotype and LDH risk. Logistic regression analysis was used to calculate the $95 \%$ confidence intervals(Cls) and odds ratios (ORs) for four different genetic models (co-dominance, dominance, recessive, log-additive) established by PLINK software (http://zzz.bwh.harvard.edu/plink/ ld.shtml) and adjusted for age and gender to estimate the risk of genotype-related lumbar disc herniation. The statistical significance is considered to be $p<0.05$ (both sides).

\section{Results}

\section{Basic information of cases and controls}

In this study, 504 patients with lumbar disc herniation and 503 healthy controls were included. Table 1 shows the characteristics of cases of lumbar disc herniation and healthy controls. There was no significant difference in age and gender between the LDH group and the healthy group ( $P>0.05)$. The average age of patients was $49.30 \pm 14.92$ years old, and that of the control group was $49.25 \pm 13.58$ years old. The primer sequences of the four sites we designed are shown in Table 2.The basic information and allele frequencies of the four SNPs (rs76107647, rs10851882, rs75393192, rs11072552) are shown in Table 3. Both SNPs in the control group were in the Hardy-Weinberg equilibrium (HWE) ( $p>0.05)$. We used chi-square test to analyze the allele distribution of the lumbar disc herniation case group and the healthy control group, and learned that the SNP rs76107647 allele A was associated with a decreased risk of lumbar disc herniation $(p=0.023, O R=0.75,95 \% \mathrm{Cl}=0.58-0.96)$.

Table 1

Characteristics of lumbar disc herniation cases and healthy controls.

\begin{tabular}{|c|c|c|c|}
\hline Characteristics & Cases (504) & Control (503) & $\mathbf{P}$ \\
\hline Age & & & 0.956 \\
\hline Mean age $\pm S D$ & $49.30 \pm 14.92$ & $49.25 \pm 13.58$ & \\
\hline Age $>49$ & $252(50 \%)$ & $251(50 \%)$ & \\
\hline Age $\leq 49$ & $252(50 \%)$ & $252(50 \%)$ & \\
\hline Gender & & & 0.970 \\
\hline Male & $294(58 \%)$ & $294(58 \%)$ & \\
\hline Female & $210(42 \%)$ & $209(42 \%)$ & \\
\hline \multicolumn{4}{|c|}{ SD: standard deviation. } \\
\hline \multicolumn{4}{|c|}{$p$ values were calculated from two-sided $x^{2}$ test. } \\
\hline $\mathrm{p} \otimes 0.05$ indicates & a statistical sig & cant. & \\
\hline
\end{tabular}


Table 2

Primer information sheet

\begin{tabular}{|llllll}
\hline SNP_ID & 2nd-PCRP & 1st-PCRP & UEP_DIR & UEP_SEQ \\
\hline rs76107647 & ACGTTGGATGGAAACCTCGTCTCTACTAAG & ACGTTGGATGTCAAGCTATTCTCCCGCATC & F & gGGTGTGCTGGCTTGC \\
rs10851882 & ACGTTGGATGACACTCCTCGTTGAAGCATC & ACGTTGGATGTGAGTTAGGGAGCTCTACAC & R & ccccaGTTGAAGCATCCCCATCTA \\
rs75393192 & ACGTTGGATGAGGGCTGAGAGTATTGGATG & ACGTTGGATGTTCCATAACCTGCCCAAGTG & R & aTCAGAGGAGCCTCTTA \\
rs11072552 & ACGTTGGATGTGTCGTGGCTGCTTGTAAAG & ACGTTGGATGTGGTGAGGAAAGGATTAGGG & R & CTGCTTGTAAAGTGAAGG \\
\hline
\end{tabular}

Table 3

Basic information and allele frequencies of the four selected SNPS

\begin{tabular}{|c|c|c|c|c|c|c|c|c|c|}
\hline \multirow[t]{2}{*}{ SNP-ID } & \multirow[t]{2}{*}{ Gene } & \multirow[t]{2}{*}{ Chromosome } & \multirow[t]{2}{*}{ Position } & \multirow[t]{2}{*}{ Alleles A/B } & \multicolumn{2}{|l|}{ MAF } & \multirow[t]{2}{*}{ HWE- $P$} & \multirow[t]{2}{*}{ OR $(95 \% \mathrm{Cl})$} & \multirow[t]{2}{*}{$P$} \\
\hline & & & & & case & control & & & \\
\hline rs76107647 & PTPN9 & 15 & 75827187 & $A / G$ & 0.125 & 0.16 & 0.74 & $0.75(0.58-0.96)$ & $0.023^{*}$ \\
\hline rs10851882 & PTPN9 & 15 & 75836279 & $\mathrm{~T} / \mathrm{C}$ & 0.52 & 0.486 & 0.475 & $1.15(0.96-1.36)$ & 0.14 \\
\hline rs75393192 & PTPN9 & 15 & 75870551 & $\mathrm{~T} / \mathrm{C}$ & 0.092 & 0.076 & 0.514 & $1.24(0.91-1.71)$ & 0.181 \\
\hline rs11072552 & PTPN9 & 15 & 75893088 & $\mathrm{C} / \mathrm{A}$ & 0.355 & 0.379 & 0.07 & $0.90(0.75-1.08)$ & 0.273 \\
\hline
\end{tabular}

SNP: Single nucleotide polymorphism; HWE: Hardy-Weinberg equilibrium; OR: Odds ratio; 95\% Cl: 95\% confidence interval.

HWE $p$-value obtained from $x^{2}$ test $(p>0.05)$.

$p$ values were calculated from Chi-squared test regarding to the allele distribution frequencies among lumbar disc herniation patients and healthy controls.

${ }^{*} p<0.05$ indicates SNP with statistical significance

\section{Genetic model analysis between PTPN9 gene and LDH risk}

The genetic relationships between the four selected single nucleotide polymorphisms and the risk of lumbar disc herniation are shown in Table 4. Our research showed that the rs76107647 in PTPN9 gene was significantly related to the risk of LDH reduction (adjusted, $A / A$ vs. G/G: $p=0.035, O R=0.33,95 \% C L=0.12-$ $0.92 ; \mathrm{A} / \mathrm{A}$ vs. $\mathrm{AG} / \mathrm{GG}: \mathrm{p}=0.044, \mathrm{OR}=0.34,95 \% \mathrm{CL}=0.12-0.97 ; \log$ - additive model $\mathrm{p}=0.022, \mathrm{OR}=0.74,95 \% \mathrm{CL}=0.58-0.96$, respectively). The rs 11072552 gene is significantly related to $\mathrm{LDH}$ risk reduction (adjusted, $\mathrm{C} / \mathrm{C}$ vs. $\mathrm{A} / \mathrm{A}: \mathrm{p}=0.048, \mathrm{OR}=0.67,95 \% \mathrm{CL}=0.45-1.00 ; \mathrm{C} / \mathrm{C}$ vs. $\mathrm{CA} / \mathrm{AA}: \mathrm{p}=0.010, \mathrm{OR}=0.62,95 \% \mathrm{CL}$ $=0.43-0.89$, respectively). 
Table 4

Genetic model analyses of four selected SNPs and the risk of lumbar disc herniation

\begin{tabular}{|c|c|c|c|c|c|c|c|c|}
\hline \multirow[t]{2}{*}{ SNP-ID } & \multirow[t]{2}{*}{ Model } & \multirow[t]{2}{*}{ Genotype } & \multirow[t]{2}{*}{ Case } & \multirow[t]{2}{*}{ Control } & \multicolumn{2}{|l|}{ Without Adjusted } & \multicolumn{2}{|l|}{ Adjusted } \\
\hline & & & & & OR $(95 \% \mathrm{Cl})$ & $p^{a}$ & OR $(95 \% \mathrm{Cl})$ & $p^{b}$ \\
\hline \multirow[t]{8}{*}{ rs76107647 } & Codominant & $\mathrm{G} / \mathrm{G}$ & 383 & 355 & 1 & & 1 & \\
\hline & & $\mathrm{A} / \mathrm{A}$ & 5 & 14 & $0.33(0.12-0.93)$ & $0.036 *$ & $0.33(0.12-0.92)$ & $0.035^{*}$ \\
\hline & & $A / G$ & 116 & 133 & $0.81(0.61-1.08)$ & 0.148 & $0.81(0.61-1.08)$ & 0.145 \\
\hline & Dominant & $\mathrm{G} / \mathrm{G}$ & 383 & 355 & 1 & & 1 & \\
\hline & & $\mathrm{AA} / \mathrm{AG}$ & 121 & 147 & $0.76(0.58-1.01)$ & 0.059 & $0.76(0.58-1.01)$ & 0.058 \\
\hline & Recessive & $A G / G G$ & 5 & 14 & 1 & & 1 & \\
\hline & & $\mathrm{A} / \mathrm{A}$ & 499 & 488 & $0.35(0.12-0.98)$ & $0.045^{*}$ & $0.35(0.12-0.97)$ & $0.044 *$ \\
\hline & Log-additive & - & - & - & $0.75(0.58-0.96)$ & $0.023^{*}$ & $0.74(0.58-0.96)$ & 0.022 * \\
\hline \multirow[t]{8}{*}{ rs10851882 } & Codominant & $\mathrm{C} / \mathrm{C}$ & 125 & 137 & 1 & & 1 & \\
\hline & & $\mathrm{T} / \mathrm{T}$ & 145 & 123 & $1.29(0.92-1.80)$ & 0.141 & $1.29(0.92-1.82)$ & 0.141 \\
\hline & & $\mathrm{T} / \mathrm{C}$ & 234 & 243 & $1.06(0.78-1.43)$ & 0.726 & $1.06(0.78-1.43)$ & 0.726 \\
\hline & Dominant & $\mathrm{C} / \mathrm{C}$ & 125 & 137 & 1 & & 1 & \\
\hline & & $\mathrm{TT} / \mathrm{TC}$ & 379 & 366 & $1.14(0.86-1.50)$ & 0.379 & $1.14(0.86-1.50)$ & 0.378 \\
\hline & Recessive & $\mathrm{TC} / \mathrm{CC}$ & 145 & 123 & 1 & & 1 & \\
\hline & & $\mathrm{T} / \mathrm{T}$ & 359 & 380 & $1.25(0.94-1.65)$ & 0.122 & $1.25(0.94-1.65)$ & 0.122 \\
\hline & Log-additive & - & - & - & $1.14(0.96-1.35)$ & 0.14 & $1.137(0.9587-1.349)$ & 0.14 \\
\hline \multirow[t]{8}{*}{ rs75393192 } & Codominant & $\mathrm{C} / \mathrm{C}$ & 416 & 431 & 1 & & 1 & \\
\hline & & $\mathrm{T} / \mathrm{T}$ & 5 & 4 & $1.30(0.35-4.86)$ & 0.701 & $1.30(0.35-4.89)$ & 0.699 \\
\hline & & $\mathrm{T} / \mathrm{C}$ & 83 & 68 & $1.27(0.89-1.79)$ & 0.186 & $1.27(0.89-1.79)$ & 0.186 \\
\hline & Dominant & $\mathrm{C} / \mathrm{C}$ & 416 & 431 & 1 & & 1 & \\
\hline & & TT/TC & 88 & 72 & $1.27(0.90-1.78)$ & 0.173 & $1.27(0.90-1.78)$ & 0.173 \\
\hline & Recessive & $\mathrm{TC} / \mathrm{CC}$ & 5 & 4 & 1 & & 1 & \\
\hline & & $\mathrm{T} / \mathrm{T}$ & 499 & 499 & $1.25(0.33-4.68)$ & 0.741 & $1.25(0.33-4.69)$ & 0.742 \\
\hline & Log-additive & - & - & - & $1.24(0.90-1.69)$ & 0.182 & $1.24(0.90-1.70)$ & 0.182 \\
\hline \multirow[t]{8}{*}{ rs11072552 } & Codominant & $\mathrm{A} / \mathrm{A}$ & 200 & 204 & 1 & & 1 & \\
\hline & & $\mathrm{C} / \mathrm{C}$ & 54 & 82 & $0.67(0.45-0.10)$ & $0.048^{*}$ & $0.67(0.45-0.10)$ & $0.048 *$ \\
\hline & & $\mathrm{C} / \mathrm{A}$ & 250 & 217 & $1.18(0.90-1.53)$ & 0.236 & $1.18(0.90-1.53)$ & 0.236 \\
\hline & Dominant & $\mathrm{A} / \mathrm{A}$ & 200 & 204 & 1 & & 1 & \\
\hline & & $\mathrm{CC} / \mathrm{CA}$ & 304 & 299 & $1.04(0.81-1.33)$ & 0.78 & $1.04(0.81-1.33)$ & 0.777 \\
\hline & Recessive & CA/AA & 54 & 82 & 1 & & 1 & \\
\hline & & $\mathrm{C} / \mathrm{C}$ & 450 & 421 & $0.62(0.43-0.89)$ & $0.01 *$ & $0.62(0.43-0.89)$ & $0.01^{*}$ \\
\hline & Log-additive & - & - & - & $0.90(0.75-1.08)$ & 0.273 & $0.90(0.75-1.08)$ & 0.273 \\
\hline \multicolumn{9}{|c|}{ SNP: Single nucleotide polymorphism; OR: Odds ratio; 95\% Cl: 95\% confidence interval. } \\
\hline \multicolumn{9}{|c|}{$p^{a}$ - value calculated by Chi-squared test } \\
\hline \multicolumn{9}{|c|}{$P^{b-}$ value calculated by Chi-squared test and adjusted by gender and age } \\
\hline
\end{tabular}

\section{Stratification analysis by age}

We also performed a stratified analysis to assess the effect of SNPs genotypes in four genetic models on the risk of lumbar disc herniation. Since the average age of the case group and the control group in our study population was 49 years, we stratified by the age of 49 years. Table 5 shows the relationship between 
Table 6 lists the relationship between SNP genotype and risk of lumbar disc herniation in gender stratification. In females, the rs76107647 was related to a decrease the risk of LDH (adjusted, A/A vs. G/G: $p=0.017, \mathrm{OR}=0.08,95 \% \mathrm{Cl}=0.01-0.64 ; \mathrm{A} / \mathrm{A}$ vs. AG/GG: $\mathrm{p}=0.019,0 \mathrm{R}=0.09,95 \% \mathrm{Cl}=0.01-0.67$; log - additive model: $p=0.018, O R=0.64,95 \% \mathrm{Cl}=0.44-0.93$, respectively). In males, however, we did not find a significant association lumbar disc herniation in any genetic model.

Table 6

Association between genotype and risk of lumbar disc herniation after gender stratification

\begin{tabular}{|c|c|c|c|c|c|c|}
\hline \multirow[t]{2}{*}{ SNP-ID } & \multirow[t]{2}{*}{ Model } & \multirow[t]{2}{*}{ Genotype } & \multicolumn{2}{|l|}{ Male } & \multicolumn{2}{|l|}{ Female } \\
\hline & & & OR $(95 \% \mathrm{Cl})$ & $\mathbf{P}$ & OR $(95 \% \mathrm{Cl})$ & $\mathbf{P}$ \\
\hline \multirow[t]{8}{*}{ rs76107647 } & Codominant & $\mathrm{G} / \mathrm{G}$ & 1 & & 1 & \\
\hline & & $\mathrm{A} / \mathrm{A}$ & $1.28(0.288-5.79)$ & 0.753 & $0.08(0.01-0.64)$ & $0.017 *$ \\
\hline & & $A / G$ & $0.80(0.54-1.17)$ & 0.251 & $0.81(0.53-1.26)$ & 0.356 \\
\hline & Dominant & $\mathrm{G} / \mathrm{G}$ & 1 & & 1 & \\
\hline & & $\mathrm{AA} / \mathrm{AG}$ & $0.82(0.56-1.19)$ & 0.293 & $0.70(0.46-1.07)$ & 0.1 \\
\hline & Recessive & AG/GG & 1 & & 1 & \\
\hline & & $\mathrm{A} / \mathrm{A}$ & $1.34(0.30-6.08)$ & 0.702 & $0.09(0.01-0.67)$ & $0.019 *$ \\
\hline & Log-additive & - & $0.85(0.60-1.21)$ & 0.375 & $0.64(0.44-0.93)$ & $0.018 *$ \\
\hline \multirow[t]{8}{*}{ rs10851882 } & Codominant & $\mathrm{C} / \mathrm{C}$ & 1 & & 1 & \\
\hline & & $\mathrm{T} / \mathrm{T}$ & $1.17(0.75-1.84)$ & 0.489 & $1.47(0.87-2.48)$ & 0.149 \\
\hline & & $\mathrm{T} / \mathrm{C}$ & $1.03(0.69-1.52)$ & 0.903 & $1.10(0.69-1.76)$ & 0.699 \\
\hline & Dominant & $\mathrm{C} / \mathrm{C}$ & 1 & & 1 & \\
\hline & & $\mathrm{TT} / \mathrm{TC}$ & 1.07(0.74-1.55) & 0.707 & $1.23(0.79-1.90)$ & 0.36 \\
\hline & Recessive & $\mathrm{TC} / \mathrm{CC}$ & 1 & & 1 & \\
\hline & & $\mathrm{T} / \mathrm{T}$ & $1.15(0.80-1.67)$ & 0.449 & $1.38(0.90-2.12)$ & 0.136 \\
\hline & Log-additive & - & $1.08(0.86-1.36)$ & 0.49 & $1.21(0.93-1.58)$ & 0.146 \\
\hline \multirow[t]{8}{*}{ rs75393192 } & Codominant & $\mathrm{C} / \mathrm{C}$ & 1 & & 1 & \\
\hline & & $\mathrm{T} / \mathrm{T}$ & & 0.999 & $0.80(0.18-3.63)$ & 0.771 \\
\hline & & $\mathrm{T} / \mathrm{C}$ & $1.07(0.66-1.73)$ & 0.782 & $1.53(0.92-2.55)$ & 0.105 \\
\hline & Dominant & $\mathrm{C} / \mathrm{C}$ & 1 & & 1 & \\
\hline & & TT/TC & $1.12(0.70-1.80)$ & 0.629 & $1.40(0.88-2.36)$ & 0.144 \\
\hline & Recessive & $\mathrm{TC} / \mathrm{CC}$ & 1 & & 1 & \\
\hline & & $\mathrm{T} / \mathrm{T}$ & & 0.999 & $0.74(0.16-3.35)$ & 0.697 \\
\hline & Log-additive & - & 1.18(0.75-1.86) & 0.486 & $1.30(0.84-2.01)$ & 0.239 \\
\hline \multirow[t]{8}{*}{ rs11072552 } & Codominant & $\mathrm{A} / \mathrm{A}$ & 1 & & 1 & \\
\hline & & $\mathrm{C} / \mathrm{C}$ & $0.72(0.43-1.21)$ & 0.216 & $0.61(0.34-1.12)$ & 0.113 \\
\hline & & $\mathrm{C} / \mathrm{A}$ & $1.20(0.85-170)$ & 0.308 & $1.14(0.75-1.73)$ & 0.534 \\
\hline & Dominant & $\mathrm{A} / \mathrm{A}$ & 1 & & 1 & \\
\hline & & $\mathrm{CC} / \mathrm{CA}$ & $1.07(0.77-1.49)$ & 0.675 & $0.99(0.67-1.46)$ & 0.944 \\
\hline & Recessive & $\mathrm{CA} / \mathrm{AA}$ & 1 & & 1 & \\
\hline & & $\mathrm{C} / \mathrm{C}$ & $0.65(0.40-1.06)$ & 0.09 & $0.57(0.33-1.00)$ & 0.051 \\
\hline & Log-additive & - & $0.94(0.74-1.19)$ & 0.582 & $0.86(0.65-1.14)$ & 0.294 \\
\hline \multicolumn{7}{|c|}{ SNP: Single nucleotide polymorphism; OR: Odds ratio; 95\% Cl: 95\% confidence interval. } \\
\hline \multicolumn{7}{|c|}{$\mathrm{p}$ values were calculated from Chi-squared test. Adjusted for age and sex in a logistic regression model. } \\
\hline & & ificanc & & & & \\
\hline
\end{tabular}

\section{Discussion}


Our study shows that rs76107647 and rs11072552 on PTPN9 are significantly associated with reduced susceptibility to lumbar disc disease. As far as we know, there are not any reports on the risks of these two SNPS. In addition, we tested the age and gender stratification of the PTPN9 and analyzed the correlation between their SNPs and LDH risks. In age stratification, rs11072552 has a protective effect on LDH risk of people over 49 years old. Rs76107647 can reduce LDH risk of people under 49 years old. In the gender stratification group, rs76107647 reduced LDH risk of female subjects. Therefore, we speculate that the PTPN9 may play a role in reducing risk factors for LDH progression.

Protein tyrosine phosphatase non receptor 9 (PTPN9), also known as PTPMeg2 or Meg2, is a cytoplasmic PTPs, which is widely expressed in brain tissues, white blood cells, endocrine cells and other tissues. It has been shown that multiple signaling pathways and physiological processes can be regulated by PTPN9[14]. In our study, we found that new genes PTPN9 affect the risk of LDH. It has been identified as protective factors of LDH. Therefore, when a disc ruptures, NGF protein levels rise in the ruptured disc, possibly leading to pathologic nerve growth to the disc. So, we believe that NGF may be the key factor leading to the discogenic pain. And PTPN9 can down-regulate NGF/TrkA signal transduction[18].Moreover, VEGF is expressed in the degenerative disc tissue in the patients with lumbar disc herniation, which acts on the formation and infiltration of new blood vessels and accelerates the degeneration of the disc tissue[19], while PTP-Meg2 negatively regulates the downstream signal transduction of vascular endothelial growth factor (VEGF) - induced receptors in endothelial cells [9]. Moreover, immune cells and nonimmune cell, such as NP cells, fibroblasts and endothelial cells, can store and secrete a variety of cytokines, such as IL-1, IL-6, TNF-a and granulocyte macrophage colony stimulating factor, which may lead to the final occurrence of sciatica[20]. Among them, IL-6 also induces the activation of JAK/STAT3 signaling pathway and plays a role in inflammation[21]. In NP tissues, IL-21 mainly induces the expression of TNF-a through JAK-STAT3 signaling pathway, which aggravates the degeneration of the intervertebral disc[22]. In addition, activation of RAGE/STAT3 pathway plays an important role in LDH persistent pain[23]. PTPN9 can dephosphorylate and inactivate vascular endothelial growth factor receptor 2 and STAT-3[14], and negatively regulate STAT3 signaling pathway[24]. Therefore, PTPN9 is a new target for the treatment of LDH.

It should be pointed out that this study has some limitations. First of all, all participants in this study are from the same hospital, and there may be selection bias in the study population. There are many factors that affect LDH susceptibility, such as lifestyle factors and environmental factors. Due to lack of information or objective reasons, we are unable to assess the impact of all factors on LDH risk. Therefore, it necessary to further studies this study.

\section{Conclusion}

In conclusion, the polymorphisms of PTPN9 in the Chinese Han population are related to the susceptibility to lumbar disc herniation, which can be seen from case-control studies. This study provides new directions for the treatment of LDH. Therefore, further comprehensive functional studies and prospective studies based on a wide population are needed to provide accurate evidence of the effect of genotype on lumbar disc herniation.

\section{List Of Abbreviations}

LDH Lumbar disc herniation

SNP Single nucleotide polymorphism

OR Odds ratio

Cl Confidence interval

MAF Minor allele frequency

HWE Hardy-Weinberg equilibrium

LD Linkage disequilibrium

\section{Declarations}

\section{Ethics approval and consent to participate}

This case-control study was conducted in accordance with the Declaration of Helsinki. And the protocol was approved by the Ethics Committee of the Affiliated Hospital of Xizang Minzu University. Written informed consents were obtained from all individuals. The procedures were in accordance with the institutional guidelines.

\section{Consent to publish}

All patients agreed to publish the manuscript.

\section{Acknowledgements}

Thank the Red Cross Hospital of Xi'an for providing the samples used in this study. We also thank the reviewers and editors for their efforts and patience. 


\section{Declaration of interest}

The authors declared no conflicts of interest in this study.

\section{Funding}

This study was funded by the Natural Science Foundation of Tibet Autonomous Region (No. XZ2019ZRG-27(Z)).

\section{Competing interests}

The authors declared no conflicts of interest. The authors alone are responsible for the content of this manuscript.

\section{Consent for publication}

Not applicable.

\section{Availability of data and materials}

The datasets supporting the conclusions of this article are included within the article and its additional file.

\section{References}

1. Yang, X., et al., CHRNA5/CHRNA3 gene cluster is a risk factor for lumbar disc herniation: a case-control study. J Orthop Surg Res, 2019. 14(1): p. 243.

2. Casa, N.L.L., et al., CASE-REPORT Association between an ACAN gene variable number tandem repeat polymorphism and lumbar disc herniation: a case control study. Genetics and Molecular Research, 2016. 15(4).

3. Liu, H., et al., A common polymorphism of COMT was associated with symptomatic lumbar disc herniation based on a large sample with Chinese Han ancestry. Scientific Reports, 2018. 8(1).

4. Jiang, H., et al., Interleukin-23 may contribute to the pathogenesis of Iumbar disc herniation through the IL-23/IL-17 pathway. J Orthop Surg Res, 2016. 11: p. 12.

5. Dong, Q., et al., Genetic polymorphisms of ALDH2 are associated with lumbar disc herniation in a Chinese Han population. Sci Rep, 2018. 8(1): p. 13079.

6. MacGregor, A.J., et al., Structural, psychological, and genetic influences on low back and neck pain: A study of adult female twins. Arthritis Care \& Research, 2004. 51(2): p. 160-167.

7. Karademir, M., O. Eser, and E. Karavelioglu, Adolescent lumbar disc herniation: Impact, diagnosis, and treatment. J Back Musculoskelet Rehabil, 2017. 30(2): p. 347-352.

8. Ji, D., et al., Correlation of EYS polymorphisms with lumbar disc herniation risk among Han Chinese population. Mol Genet Genomic Med, 2019. 7(9): p. e890.

9. Hao, Q., et al., Tyrosine phosphatase PTP-MEG2 negatively regulates vascular endothelial growth factor receptor signaling and function in endothelial cells. Am J Physiol Cell Physiol, 2012. 303(5): p. C548-53.

10. Gu, et al., Cloning and expression of a cytosolic megakaryocyte protein-tyrosine-phosphatase with sequence homology to retinaldehyde-binding protein and yeast SEC14p. Proceedings of the National Academy of Sciences, 1992.

11. Yuan, T., et al., Protein-tyrosine phosphatase PTPN9 negatively regulates ErbB2 and epidermal growth factor receptor signaling in breast cancer cells. J Biol Chem, 2010. 285(20): p. 14861-70.

12. Zhu, J., et al., PTPN9 promotes cell proliferation and invasion in Eca109 cells and is negatively regulated by microRNA-126. Oncol Lett, 2017. 14(2): $\mathrm{p}$. 1419-1426.

13. Hu, B., et al., Downregulated Expression of PTPN9 Contributes to Human Hepatocellular Carcinoma Growth and Progression. Pathol Oncol Res, 2016. 22(3): p. 555-65.

14. Wang, D., et al., PTPN9 induces cell apoptosis by mitigating the activation of Stat3 and acts as a tumor suppressor in colorectal cancer. Cancer Manag Res, 2019. 11: p. 1309-1319.

15. Li, W.T., et al., MiR-613 promotes cell proliferation and invasion in cervical cancer via targeting PTPN9. Eur Rev Med Pharmacol Sci, 2018. 22(13): p. 41074114.

16. Liu, Z., et al., MEG2 is regulated by miR-181a-5p and functions as a tumour suppressor gene to suppress the proliferation and migration of gastric cancer cells. Mol Cancer, 2017. 16(1): p. 133.

17. Wang, Y., et al., Tyrosine phosphatase MEG2 modulates murine development and platelet and lymphocyte activation through secretory vesicle function. J Exp Med, 2005. 202(11): p. 1587-97.

18. Zhang, D., et al., The Protein Tyrosine Phosphatase MEG2 Regulates the Transport and Signal Transduction of Tropomyosin Receptor Kinase A. J Biol Chem, 2016. 291(46): p. 23895-23905. 
19. Lu, X.-Y., et al., Expression and significance of VEGF and p53 in degenerate intervertebral disc tissue. Asian Pacific Journal of Tropical Medicine, 2013. 6(1): p. 79-81.

20. Martino, A.D., L. Merlini, and C. Faldini, Autoimmunity in intervertebral disc herniation: from bench to bedside. Expert Opinion on Therapeutic Targets, 2013. 17(12): p. 1461-1470.

21. Osuka, K., et al., Expression of the JAK/STAT3/SOCS3 signaling pathway in herniated lumbar discs. Neurosci Lett, 2014. 569: p. 55-8.

22. Chen, B., et al., IL-21 Is Positively Associated with Intervertebral Disc Degeneration by Interaction with TNF-a Through the JAK-STAT Signaling Pathway. Inflammation, 2017. 40(2): p. 612-622.

23. Zhang, X.S., et al., Activation of the RAGE/STAT3 Pathway in the Dorsal Root Ganglion Contributes to the Persistent Pain Hypersensitivity Induced by Lumbar Disc Herniation. Pain Physician, 2017. 20(5): p. 419-427.

24. Kim, M., et al., Protein Tyrosine Phosphatases as Potential Regulators of STAT3 Signaling. Int J Mol Sci, 2018. 19(9). 